\title{
PROBLEMA Y EJEMPLO: Los nueve cuadrados y la transformación del edificio fundacional de la Cooper Union
}

\author{
Estrella Cobo, Lisseth Mireya \\ PROBLEMA Y EJEMPLO: Los nueve cuadrados y la transformación del edificio fundacional de la Cooper Union \\ Estudios del Hábitat, vol. 18, núm. 1, 2020 \\ Universidad Nacional de La Plata, Argentina
}

Atribución no comercial compartir igual (CC BY-NC-SA) 4.0 


\section{PROBLEMA Y EJEMPLO: Los nueve cuadrados y la transformación del edificio fundacional de la Cooper Union}

PROBLEM AND EXAMPLE: The Nine-Square and The Cooper Union Foundation Building Transformation

Lisseth Mireya Estrella Cobo

Escuela Técnica Superior de Arquitectura de Madrid.

Universidad Politécnica de Madrid, España

lisseth.estrella.cobo@alumnos.upm.es
Recepción: 24 Febrero 2020

Aprobación: 19 Mayo 2020

Publicación: 30 Junio 2020

Recepción: 24 Febrero 2020

Aprobación: 19 Mayo 2020

Publicación: 30 Junio 2020

\section{Resumen:}

Enseñar con el ejemplo es parte fundamental de la estrategia pedagógica de John Hejduk. Sin mayores explicaciones, Hejduk relaciona su proyecto de transformación del edificio fundacional de la Cooper Union con el problema de los nueve cuadrados. Es un caso singular porque el edificio, lugar de la escuela de arquitectura, presenta a sus alumnos el ejemplo del problema utilizado como introducción a la disciplina misma. Con información de archivo y referencias de personas que formaron parte de la escuela en este período - John Hejduk, Peter Eisenman, Rafael Moneo y Lorna McNeur - el artículo identifica que tanto problema como ejemplo comparten la misma estructura y define el método para relacionarlos: utilizar los nueve cuadrados como herramienta de análisis aplicando los conceptos de centro-periferia, fluidez-contención y estructura-plemento. El resultado del trabajo ha llevado a comprender el contenido analítico del problema en relación con las herramientas del arquitecto: el paso de la planta a la sección en relación con los conceptos de espacio y tiempo.

Palabras CLAVE: John Hejduk, estructura, análisis, pedagogía, proyectos arquitectónicos.

\section{Abstract:}

Teaching by example is a fundamental aspect of John Hejduk's pedagogical strategy. Without further explanations, Hejduk relates his project for the interior transformation of the Cooper UnionFoundation Building to the Nine-Square problem. It is a singular case because the building, home of the school of architecture, presents to its students the example of the problem used to introduce the discipline itself. With archival information and references from people that were part of the school at that time - John Hejduk, Peter Eisenman, Rafael Moneo, and Lorna McNeur - the article identifies that both problem and example share the same structure and defines the method to relate them: to use the Nine-Square as an analytical tool applying the concepts of center-periphery, fluidity-containment and structure-panel. The paper outcome has led to an understanding of the problem's analytical content in relation to the architect's tools: the passage from plan to section in relation to the concepts of space and time.

KEYWORDS: John Hejduk, structure, analysis, pedagogy, architectural design .

\section{INTRODUCCIÓN}

COOPER ESTÁ RELACIONADA CON TEXAS - está basada en el problema de los nueve cuadrados. Es un dispositivo de enseñanza, diseñado en uno de los problemas fundamentales propuestos en la escuela. Y los alumnos están aquí, trabajando en este problema dentro de este edificio, que es un ejemplo de este problema. (Hejduk, 1985, p. 70)

El nombre de John Hejduk (1929-2000), "la figura definitiva en el campo de la pedagogía de arquitectura de la segunda mitad del siglo XX” (Moneo, 2009, p. 73), está indeleblemente ligado con la Cooper Union. No sólo hizo los tres primeros años de su formación como arquitecto allí (1947-1950), sino que regresó 14 años después para quedarse por 36 años (1964-2000), 10 como director del Departamento de Arquitectura y 25 como el primer decano de la Irwin S. Chanin School of Architecture. Fue él quien puso a la Cooper Union en el mapa con la exhibición en el Museum of Modern Art (MoMA) de Nueva York: Education of an Architect: A Point of View 1964-1971. Tanto la exhibición como el catálogo "produjeron una profunda impresión y, 

EDIF...

desde entonces, la Cooper Union fue relacionada con la nueva avant-garde que hizo su aparición en Nueva York a principios de los setentas" (Anónimo, 1980, p. 64).

En la cita introductoria, Hejduk deja claro que enseñar a través del ejemplo es fundamental en su pedagogía. Rafael Moneo (1980) en su artículo La obra de John Hejduk o la pasión por enseñar es el único que hasta ahora ha investigado en esa dirección al relacionar los problemas que Hejduk propuso en la Cooper Union con su obra. Moneo explica que esta última puede ser considerada material de enseñanza porque es la demostración práctica de los principios que Hejduk profesa y, además, es el material que los alumnos tienen disponible para comprender la teoría que nunca se llega a expresar claramente. A partir de esta premisa, Moneo relaciona el problema de los nueve cuadrados con la serie Texas Houses. Por su parte, el ejemplo de la transformación del edificio de la Cooper Union es singular porque confluyen en un mismo lugar y en un mismo marco temporal problema y ejemplo. El edificio, lugar de la escuela de arquitectura, presenta a los alumnos el ejemplo del problema utilizado para introducir la disciplina en el espacio dedicado a la educación del arquitecto.

A pesar de su relevancia hasta ahora no existe literatura que relacione los puntos que Hejduk señala: la transformación del edificio de la Cooper Union (1969-1974), la serie Texas Houses (1954-1962) y el problema de los nueve cuadrados (1954-1978). El objetivo de esta investigación es descubrir parte del contenido didáctico del singular dispositivo de enseñanza que Hejduk ofreció a sus alumnos con la transformación del edificio fundacional de la Cooper Union.

\section{Método}

Con 40 años cumplidos, Hejduk (1996) fue muy consciente de la envergadura que el encargo de la transformación interior del edificio fundacional de la Cooper Union representaba; él sabía que se desenvolvía en un medio que lo veía como un buen profesor y teórico pero que no ha construido mucho. Hasta 1969, año en el que empezó a trabajar en el proyecto de la transformación, había terminado la serie TexasHouses (1954-1962) y la serie Diamond (1962-1967) mientras la serie Wall House (1964-1974) estaba en su fase inicial. En paralelo con estos proyectos, Hejduk escribe dos artículos que hacen patente la influencia de Le Corbusier en su obra y completan el contexto de los temas que había investigado hasta entonces. El primero titulado Out of Time into Space $(1965)^{1}$ es un análisis del Carpenter Center de Le Corbusier y está relacionado con la serie Diamond. Uno de los temas que investiga aquí es la relación entre frontalidad y rotación, la concepción del espacio en relación con la posición del observador. En el segundo artículo titulado Centralized Relief upon a Tableau (ca. 1969), Hejduk expone la relación entre la Cité de Refuge (1929-1933) y el Pabellón Suizo (1930-32) de Le Corbusier con su proyecto $3 / 4$ House (1966-1968) haciendo evidente la genealogía cubista derivada de Le Corbusier. Las ideas contenidas en este artículo son posteriormente expresadas en la serie $W$ all House a través de la cual estudia y expresa el concepto de tiempo en arquitectura.

En este contexto, el proyecto de la Cooper Union (1969-1974) corresponde al período en el que completa la serie Wall House. Por su parte, el problema de los nueve cuadrados (1954-1978) fue concebido y propuesto por primera vez cuando con 25 años se inicia como profesor en la Universidad de Austin, Texas. De ahí se deriva el nombre, Texas Houses, en referencia al lugar y en cierto sentido también a la experiencia que vivió allí con el grupo de personas que inspiraron y acompañaron esta etapa de su carrera. Hejduk (1985) dedica esta serie a Colin Rowe y Robert Slutzky, ${ }^{2}$ hombres de intelecto y creación. De acuerdo con Moneo (1980), las primeras casas de esta serie "nos permiten entender con claridad y precisión lo que él [Hejduk] propuso en el problema de los nueve cuadrados" (p. 75). Es importante comprender el ejemplo de la transformación del edificio de la Cooper Union dentro de este contexto porque la obra de Hejduk se caracteriza por su continuidad, cada nuevo proyecto parece estar directamente relacionado con el anterior (Moneo, 1980) y, en particular, la transformación de la Cooper Union y la serie Wall House constituyen el cierre de la etapa que inicia en 1954. 
Ahora bien, para identificar cuál es la relación entre el problema de los nueve cuadrados y la transformación del edificio de la Cooper Union se ha reconstruido la historia de lo que se conocía en la escuela acerca de la transformación y del problema y se ha recopilado documentación de archivo, bibliografía, entrevistas y artículos de profesores y alumnos ${ }^{3}$ que formaron parte de la escuela en el período en que el problema fue propuesto (1964-1978). Peter Eisenman (1979) escribe sobre las Texas Houses, Lorna McNeur (1983) sobre el problema de los nueve cuadrados y Rafael Moneo (1980) pone ambos en relación. Los dos primeros coinciden en el carácter reduccionista de Hejduk que tiene el objetivo de producir una esencia compleja. Condición que en la obra de Hejduk tiene su origen en 1954 cuando empezó a enseñar. McNeur (1983) añade que el problema de los nueve cuadrados es una herramienta de análisis que permite comprender el orden subyacente del proyecto (p. 2). Con estos fundamentos, los nueve cuadrados se utilizaron para analizar la transformación de la Cooper Union y descubrir parte de su contenido didáctico.

\section{EL EDIFICIO FUNDACIONAL DE LA COOPER UNION Y SUS TRANSFORMACIONES}

The Cooper Union for the Advancement of Science and Art es una institución de pequeña escala y muy selectiva. ${ }^{4}$ Fundada en 1859 por la férrea decisión de Peter Cooper de ofrecer "educación gratuita a quien lo merezca" (The Cooper Union, 1970, p. 3). ${ }^{5}$ La creación de la Cooper Union y de su primer edificio fueron de los proyectos más importantes de su vida. Es más, dos de las innovaciones presentes en el edificio ${ }^{6}$ diseñado por Frederich A. Peterson son ideas de Peter Cooper. Hejduk (1996) explica que Peter Cooper transformó los rieles del tren - acero laminado en su fábrica de Nueva Jersey - en un elemento estructural dentro del edificio al modificar la forma de la sección. Además, "el edificio se diseñó para dar cabida a un elevador prácticamente antes de que esta invención haya estado disponible para el uso de pasajeros" (New York City Landmarks Preservation Committee, 1966). "Peter Cooper sabía [que] el elevador para pasajeros se inventaría. (...) apostó por el círculo porque es la forma más eficiente, espacialmente, de poner la mayor cantidad de personas en el pequeño pozo y transportarlos verticalmente" (O’Donnell, Elizabeth. como se citó en Scott, 2019). Finalmente, el cuadrado fue la forma adoptada por la industria para el elevador y la primera cabina que se instaló fue cuadrada. 

EDIF...

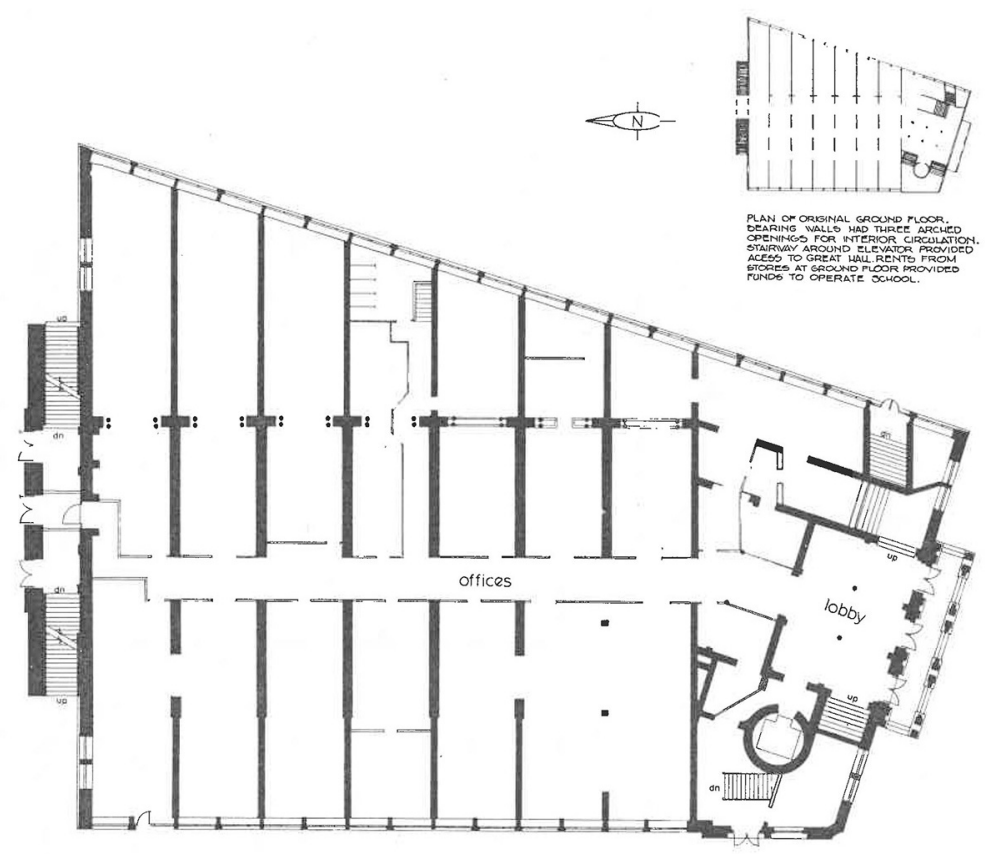

ground floor plan

FIGURA 1

LEVANTAMIENTO DE LA PLANTA BAJA ANTES DE LA TRANSFORMACIÓN DE HEJDUK

Historic American Engineering Record. (1971). Cooper Union Foundation Building Project:

Ground Floor Plan [plano]. Washington D.C.: Library of Congress Prints and Photographs Division

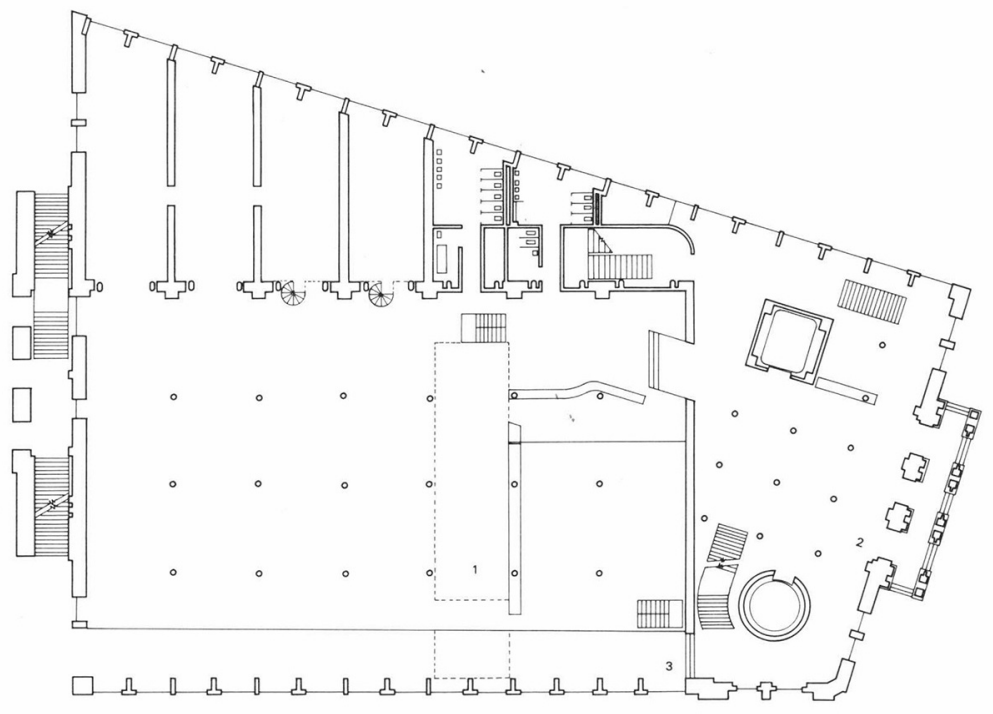

FIGURA 2

PLANTA BAJA DE LA PROPUESTA DE HEJDUK

FUENTE: HEJDUK, J. (1970). THE COOPER UNION RENOVATION PROJECT, P. 74

En 1969, siete años después de haber sido designado patrimonio de la ciudad de Nueva York y con 110 años a sus espaldas, el edificio tenía "problemas estructurales, de carga, de seguridad frente a incendios, etc.” (Hejduk, 1996, 12). Una renovación integral fue necesaria. ${ }^{7}$ Hejduk junto con el ingeniero Peter 
Bruder, también profesor de la Cooper Union, trabajaron en conjunto en el proyecto. Llama la atención la decisión de Hejduk de adscribirse a las reglas del juego de la estructura original del edificio porque decide reconstruir el orden estructural preexistente en una zona del edificio que fue totalmente derrocada. A este respecto, Kenneth Frampton (1975) al valorar el proyecto, recalca que uno de los aspectos más notables en cualquier acto de conversión es la forma en que la morfología fundamental de la estructura original se mantiene indeleble. Frampton aclara que en la transformación del edificio fundacional de la Cooper Union, tanto el caparazón original como el orden estructural y espacial se mantienen. En este sentido, el valor de la intervención de Hejduk está en su capacidad para identificar la estructura original y trabajar dentro de los límites definidos por ésta hasta lograr transformarla en algo más.

Por su parte, la estructura del proyecto responde a la geometría irregular del solar estableciendo dos retículas con orientación, dimensiones y uso distinto (Figuras 1 y 2). La primera en el extremo sur contiene los vestíbulos y las circulaciones verticales. La segunda, tiene una estructura de luz mayor que corresponde a la longitud de fabricación de los rieles del tren - 5.3 metros - y configura un gran espacio rectangular de loft. Gran parte del edificio está soportada por columnas de hierro fundido. La excepción más evidente son las dos primeras plantas de la zona norte que están soportadas por muros de carga porque estaban destinadas a comercio y oficinas.

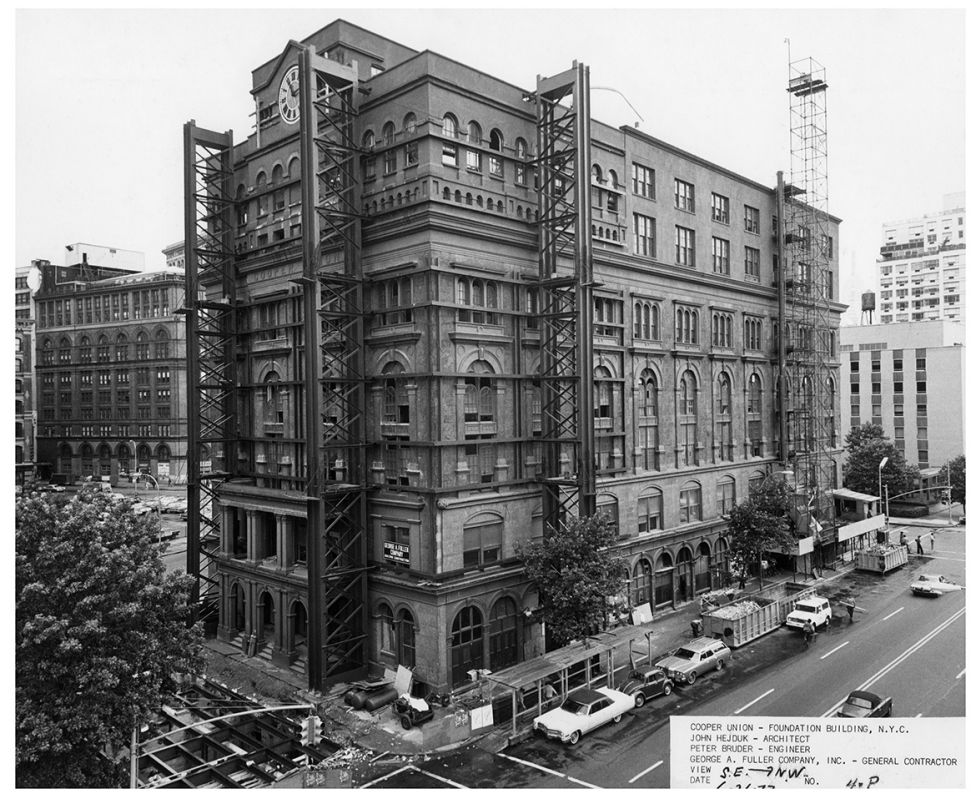

FIGURA 3

EDIFICIO FUNDACIONAL DE LA COOPER UNION EN OBRAS

HEJDUK, J., ARQUITECTO; BRUDER, P., INGENIERO \& GEORGE A. FULLER COMPANY, INC., CONTRATISTA GENERAL. FOTOGRAFÍA DE GEORGE A. FULLER COMPANY, INC. (1973). COOPER UNION FOUNDATION BUILDING INTERIOR RENOVATION [FOTOGRAFÍ]. NUEVA YORK: CORTESÍA THE IRWIN S. CHANIN SCHOOL OF ARCHITECTURE ARCHIVE, THE COOPER UNION 


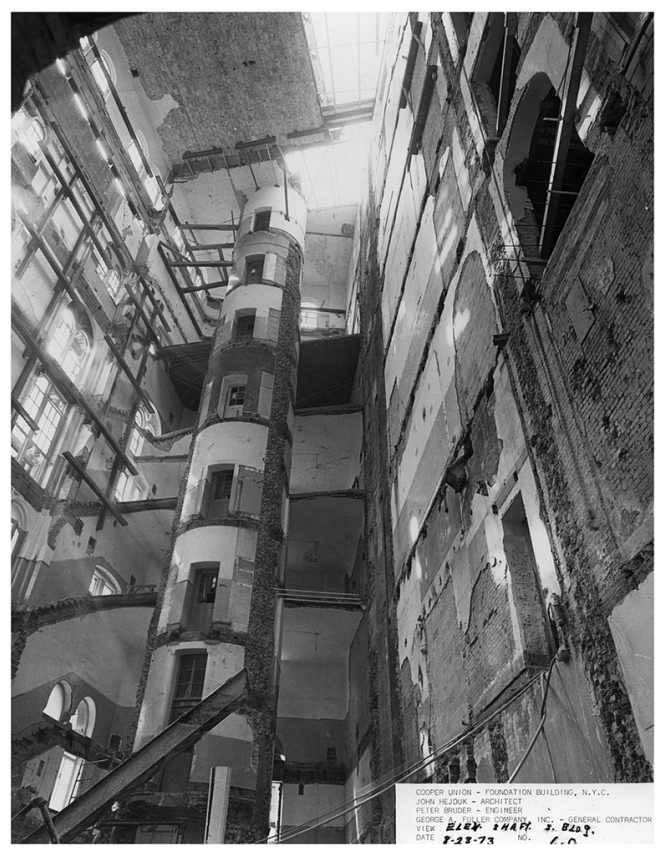

FIGURA 4

ZONA SUR DEL EDIFICIO DE LA COOPER UNIONEN OBRAS

HEJDUK, J., ARQUITECTO; BRUDER, P., INGENIERO \& GEORGE A. FULLER COMPANY, INC., CONTRATISTA GENERAL. FOTOGRAFÍA DE GEORGE A. FULLER COMPANY, INC. (1973). COOPER UNION FOUNDATION BUILDING INTERIOR RENOVATION [FOTOGRAFÍA]. NUEVA YORK: CORTESÍA THE IRWIN S. CHANIN SCHOOL OF ARCHITECTURE ARCHIVE, THE COOPER UNION.

En términos pragmáticos, la intervención de Hejduk consistió en reemplazar los muros portantes que compartimentaban el espacio de las dos primeras plantas de la zona norte por columnas (Figura 1 y Figura 2). Este cambio permitía aplicar el concepto de planta libre en todos los pisos. Hejduk explica que para lograrlo tuvieron que asegurar el edificio con cerchas verticales (Figura 3) y tuvieron que elevar 3.2 milímetros cada piso, una sección a la vez hasta lograr liberar la carga de los muros originales e "instalar debajo una nueva estructura de acero para soportar el edificio" (Hejduk, 1996, pp.12-13). Por el contrario, la zona sur se demolió completamente (Figura 4). El único elemento que dejó en pie fue el cilindro del elevador de Peter Cooper. A pesar de que esta zona estaba planteada como área de exposición y requeriría en términos pragmáticos una planta lo más libre posible, Hejduk decide volver a construir las columnas que configuraban el vestíbulo del edificio de 1859: una estructura formada por nueve cuadrados (Figura 5 y Figura 6).

Existe una aparente contradicción entre estas decisiones de proyecto. Se emplean todos los recursos materiales y técnicos para liberar la planta en la zona norte del edificio, mientras en la zona sur donde la posibilidad de eliminar las columnas era más sencilla y factible porque el interior se había demolido por completo, Hejduk prioriza la reconstrucción de la estructura preexistente. En realidad, no existe contradicción porque Hejduk está atendiendo al programa de la educación del arquitecto. Él se plantea a sí mismo el problema de los nueve cuadrados en el vestíbulo de la Cooper Union y completa así la investigación que inició con las Texas Houses en 1954. Problema y ejemplo se complementan, son dos estructuras análogas, dos manifestaciones distintas que comparten los mismos principios y se ponen a disposición de los alumnos para ser estudiadas. 


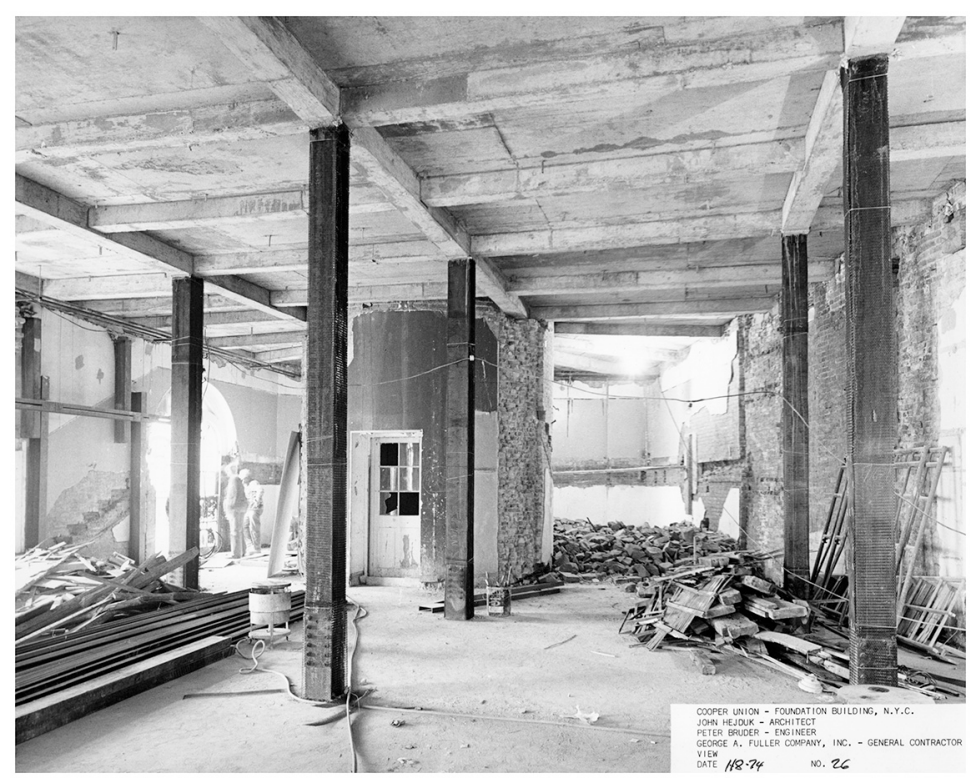

FIGURA 5

ESTRUCTURA RECONSTRUIDA EN EL VESTÍBULO DE LA COOPER UNION (1974) HEJDUK, J., ARQUITECTO; BRUDER, P., INGENIERO \& GEORGE A. FULLER COMPANY, INC., CONTRATISTA GENERAL. FOTOGRAFÍA DE GEORGE A. FULLER COMPANY, INC. (1974). COOPER UNION FOUNDATION BUILDING INTERIOR RENOVATION [FOTOGRAFÍA]. NUEVA YORK: CORTESÍA THE IRWIN S. CHANIN SCHOOL OF ARCHITECTURE ARCHIVE, THE COOPER UNION.

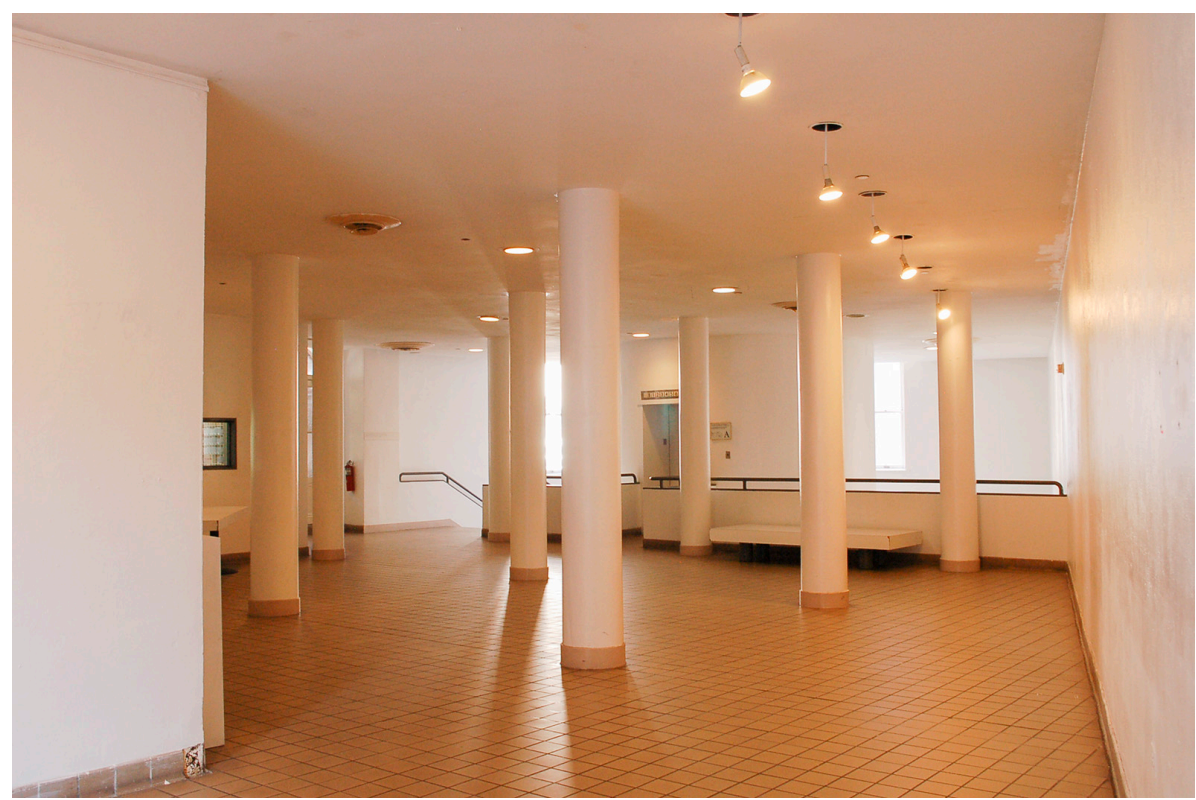

FIGURA 6

ESTRUCTURA RECONSTRUIDA EN EL VESTÍBULO DE LA COOPER UNION(2019) ELABORACIÓN PROPIA. (2019)

\section{La PEDAgogía de John HeJduK y EL PROBlema de los NUEve CuAdrados}

En el caso de Hejduk "obra y pedagogía son dos términos incuestionables y necesariamente complementarios (Lotus, 1980, p. 64). Moneo (1980) fue el primero y hasta ahora ha sido el único en escribir sobre estas relaciones indicando que en la obra se encuentra condensada la teoría que nunca se llega a expresar claramente 

EDIF...

a los alumnos. La obra es el material de enseñanza donde se manifiestan los principios que profesa. Esta posición parte de una "estrategia pedagógica deliberadamente indirecta" que tiene como propósito "fomentar la independencia intelectual de sus estudiantes. Sus enseñanzas requieren interpretación, sus ideas salir a la superficie." (Diller, Elizabeth. como se citó en Crosbie, 1988, p. 19). McNeur (1983), explica que, en el caso del problema de los nueve cuadrados, la creencia de Hejduk de que "el aprendizaje ocurre por osmosis nos forzó a cuestionar, buscar y explorar en innumerables direcciones para descubrir las respuestas que estaban escondidas en este objeto misterioso, llamado los nueve cuadrados" (p. 24).

Parte fundamental de su estrategia pedagógica indirecta incluye señalar los ejemplos. ${ }^{8}$ Existe una coincidencia temporal entre el período de aplicación del problema (1964-1978) y el período de diseño y construcción de la renovación (1969-1974). En la pequeña comunidad de estudiantes de arquitectura máximo 200 alumnos en total en la década de los 70- la historia sobre la decisión de Hejduk de reconstruir los nueve cuadrados en el vestíbulo del edificio de la Cooper Union era por todos conocida, así como su vinculación con el problema de los nueve cuadrados (W. Schwartz, comunicación personal, 15 de noviembre 2018). Es más, varios de sus alumnos colaboraron con él en el proyecto. Durante la construcción (1973-1974) "no se suspendieron las clases" (At Cooper, 1972, p. 45) y el momento más dramático fue el "hallazgo de la catedral". "La nave se formó cuando todos los pisos en el extremo sur se eliminaron por completo. (...) Cuando todo estuvo despejado, los trabajadores (...) se abrieron paso entre estudiantes, profesores y prensa (...) para comenzar la construcción del acero” (Yee, 1974, p. 100). Estos hechos evidencian que las referencias para relacionar los nueve cuadrados con el vestíbulo de la Cooper Union estaban a disposición de los alumnos. 


\subsection{El problema de los nueve cuadrados}

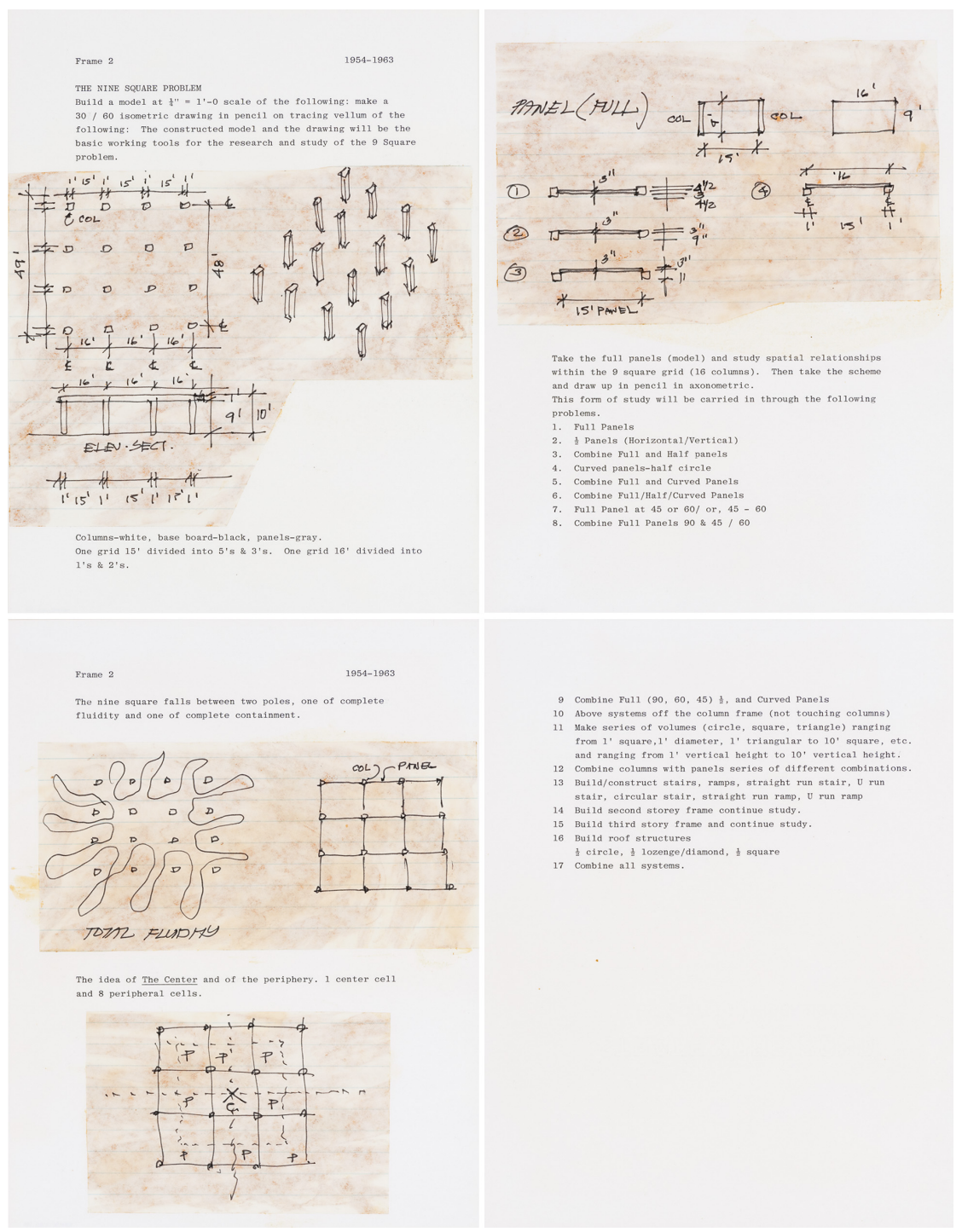

FIGURA 7

ENUNCIADO DEL PROBLEMA DE LOS NUEVE CUADRADOS

HEJDUK, J. (CA. 1963-1985). CONCEPTUAL DRAWINGS WITH NOTES FOR THE NINE SQUARE PROBLEM [ENUNCIADO]. MONTREAL: CANADIAN CENTRE FOR ARCHITECTURE

El problema de los nueve cuadrados es utilizado como una herramienta pedagógica en la introducción de la arquitectura a los nuevos estudiantes. Trabajando dentro del problema el estudiante empieza a descubrir y a comprender los elementos de la arquitectura. Retícula, estructura, columna, viga, panel, centro, periferia, campo, borde, línea, plano, volumen, extensión, compresión, tensión, desplazamiento, etc. El estudiante empieza a probar el significado de la planta, la elevación, la sección y los detalles. Él aprende a dibujar. Él empieza a comprender las relaciones entre los dibujos bidimensionales, proyecciones axonométricas y la forma tridimensional (modelos). El estudiante estudia y dibuja su esquema en planta y en axonometría y busca las implicaciones tridimensionales en el modelo. Una comprensión de los elementos es revelada, una idea de fabricación surge. (Hejduk, 1971, p. 7)

La información que existe sobre el problema de los nueve cuadrados escrita por Hejduk es puntual: el enunciado previo a su trabajo en la Cooper Union como profesor (1954-1963) que está en el Canadian Centre for Architecture (Figura 7), las notas sobre el problema (ca. 1970) que se encuentran en el archivo de arquitectura de la Cooper Union y la descripción del problema incluida en el catálogo de la exhibición del 

EDIF...

MoMA (1971). A pesar de que ninguna de ellas contiene una explicación exhaustiva del problema, el estudio de los tres documentos muestra que existen principios que se han mantenido a lo largo del tiempo.

Por un lado, la descripción del problema que encabeza esta sección es una escueta y precisa definición de objetivos. Hejduk, a más de hacer una lista de los elementos que el alumno empieza a descubrir y comprender, señala la importancia del dibujo y de los modelos como herramientas básicas de trabajo dentro del problema. Por lo tanto, están estudiando lo que implica y significa en arquitectura el paso de las dos a las tres dimensiones. Específicamente el paso de los dibujos bidimensionales -planta, sección, elevación y detalles - a las tres dimensiones del modelo. En este contexto las proyecciones axonométricas son elementos duales que no pertenecen ni al uno ni al otro. Hejduk en el enunciado (Figura 7) propone instrucciones precisas tanto para el modelo como para los dibujos porque es consciente de que la concepción y el uso de estas herramientas está cargada de significado. Por lo tanto, es a través de las actividades propuestas por el ejercicio que el alumno llega a comprender su contenido. El aprender a dibujar y hacer modelos plantea para cada alumno una interrogante que va más allá del valor instrumental de estas herramientas, están empezando a probar las relaciones entre las dos y las tres dimensiones, descubriendo sus implicaciones y significados.

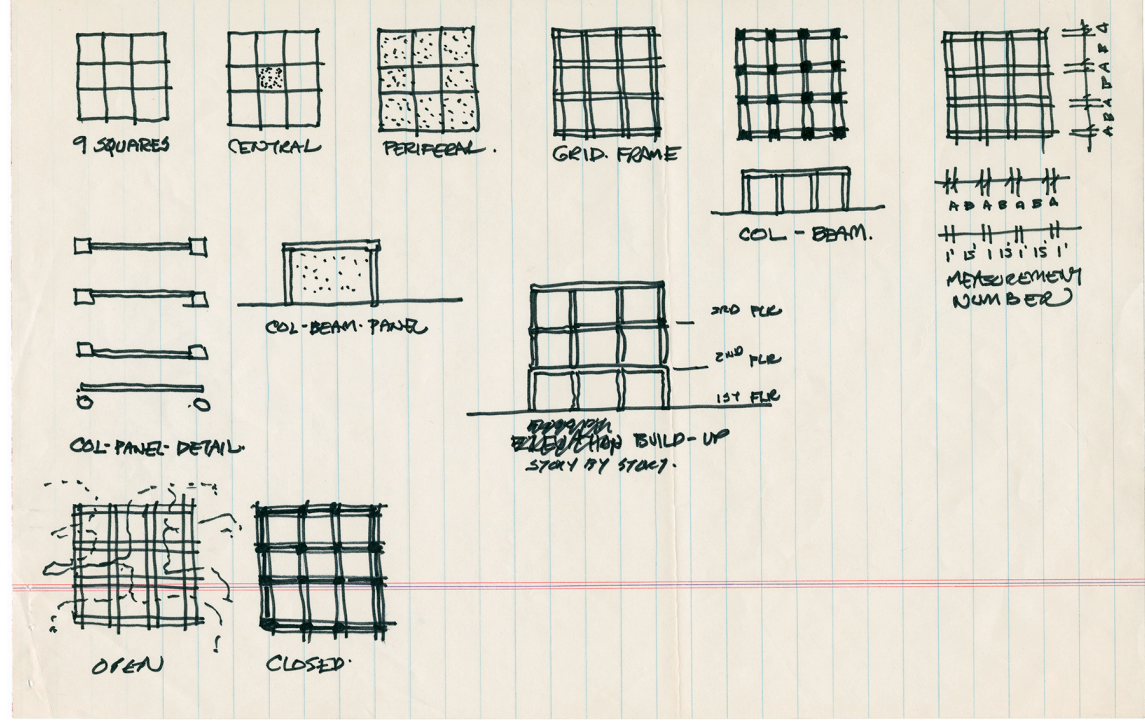

FIGURA 8

DESCRIPCIÓN GRÁFICA DE LOS INGREDIENTES DE LOS NUEVE CUADRADOS. HEJDUK, J. (CA. 1970). NINE SQUARE GRID DIAGRAM [NOTAS]. NUEVA YORK: CORTESÍA THE IRWIN S. CHANIN SCHOOL OF ARCHITECTURE ARCHIVE, THE COOPER UNION.

Por otro lado, Hejduk en las notas hace una síntesis de lo que ha sido el problema hasta la fecha. Explica que el problema "ha sido usado por diferentes instructores quienes lo han adaptado a su propia metodología de enseñanza" y, además, "ha atravesado una serie de evoluciones y metamorfosis; sin embargo, ha mantenido sus características esenciales". Como parte de la definición de estas últimas, incluye una descripción gráfica de lo que él denominó "algunos de los ingredientes" del problema (Figura 8). Esta descripción es una lista de relaciones: nueve cuadrados, uno central, ocho en la periferia para ejemplificar la relación entre centro y periferia; columna-panel-detalle, columna-viga-panel y abierto-cerrado para identificar cómo la relación entre estructura portante y plemento puede generar cualidades espaciales opuestas: fluidez-contención y, finalmente, retícula-estructura, columna-viga, medida-número y construir piso por piso para describir las relaciones que se producen en la estructura.

Los conceptos de centro-periferia, fluidez-contención y estructura-plemento expresados en las notas están también incluidos en el enunciado del problema (1954-1963) (Figura 7). Hejduk escribe: "Los nueve cuadrados están entre dos polos, uno de fluidez completa y otro de contención completa" y añade: "La idea 
de Centro y de periferia. 1 celda central y 8 celdas perimetrales". La relación estructura-plemento, uno de los problemas básicos de la arquitectura, está expresada en las acciones propuestas por el problema. Hejduk da las siguientes instrucciones: "tome los paneles completos (modelo) y estudie relaciones espaciales dentro de la retícula de los nueve cuadrados (16 columnas)". Donde los paneles son los elementos no portantes - plemento - y la estructura son las 16 columnas. Esta forma de estudio se mantiene a través de una serie de problemas que progresiva y sistemáticamente incorporan distintas configuraciones de elementos no portantes. ${ }^{9}$ Es más, tanto en el enunciado como en las notas Hejduk dibuja cuatro formas de relacionar el panel con la estructura portante haciendo evidente los problemas constructivos estudiados.

Hasta ahora, la única explicación desarrollada desde dentro de la Cooper ha sido la de McNeur (1983). Ella explica que el problema de los nueve cuadrados es un instrumento de análisis que permite comprender el orden subyacente del proyecto porque es una "estructura reduccionista" que "encarna los conceptos, órdenes y elementos fundamentales de la arquitectura" y tiene el objetivo de "alentar al estudiante a llegar a una comprensión clara y profunda de los eventos singulares que constituyen el altamente complejo organismo de la arquitectura". Señala además que pocas personas reconocen en los nueve cuadrados una estructura reduccionista y justamente por esta razón, fallan en comprender su potencial como herramienta de diseño clásica.

\section{RELACIONES ENTRE EL PROBLEMA DE LOS NUEVE CUADRADOS Y LA TRANSFORMACIÓN DEL EDIFICIO DE LA COOPER UNION}

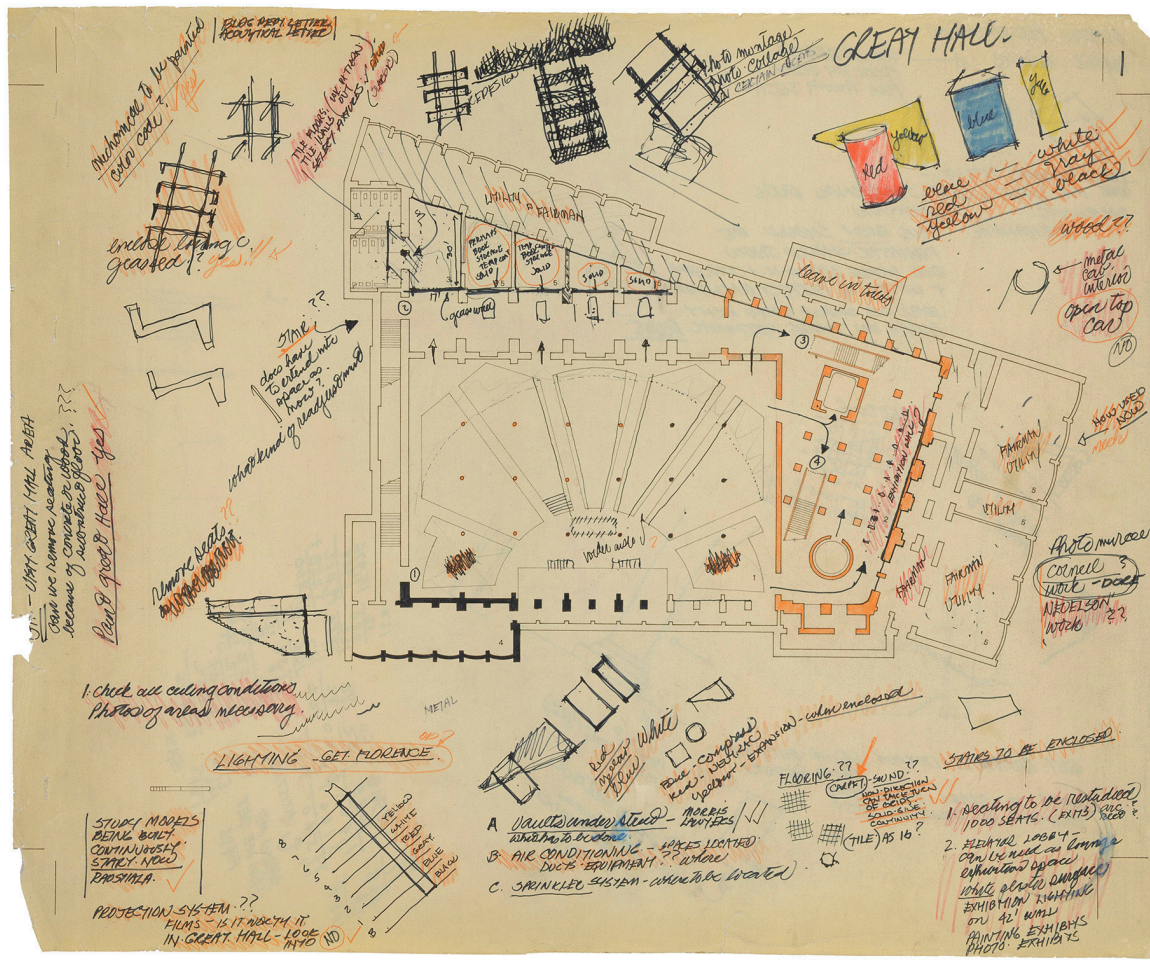

FIGURA 9

TRANSFORMACIÓN INTERIOR DEL EDIFICIO FUNDACIONAL DE

LA COOPER UNION: PLANTA SÓTANO DEL PROCESO DE DISEÑO

HEJDUK, J. (1972). COOPER UNION FOUNDATION BUILDING INTERIOR RENOVATION, LOWER

LEVEL DESIGN DEVELOPMENT PLAN. [PLANO DE TRABAJO]. NUEVA YORK: CORTESÍA

THE IRWIN S. CHANIN SCHOOL OF ARCHITECTURE ARCHIVE, THE COOPER UNION. 

EDIF...

Con el objetivo de identificar las relaciones entre el problema y el ejemplo se utilizaron los nueve cuadrados como herramienta de análisis aplicando los conceptos de centro-periferia, fluidez-contención y estructuraplemento. Para conocer el proceso de diseño del proyecto se estudiaron los planos de trabajo del proyecto de transformación del edificio de la Cooper (ca. 1970) conservados en el archivo de arquitectura de la escuela.

En la planta sótano del proyecto de transformación de la Cooper Union(Figura 9), Hejduk codifica con una forma y color los elementos que pondrá en relación dentro de la estructura de los nueve cuadrados. Círculo rojo para el elevador de Peter Cooper, cuadrado azul para el nuevo elevador y triángulo amarillo para los planos verticales. Inicia el proyecto haciendo una clara referencia a la relación entre estructura y plemento donde las partes a insertar son la definición de este problema en particular.

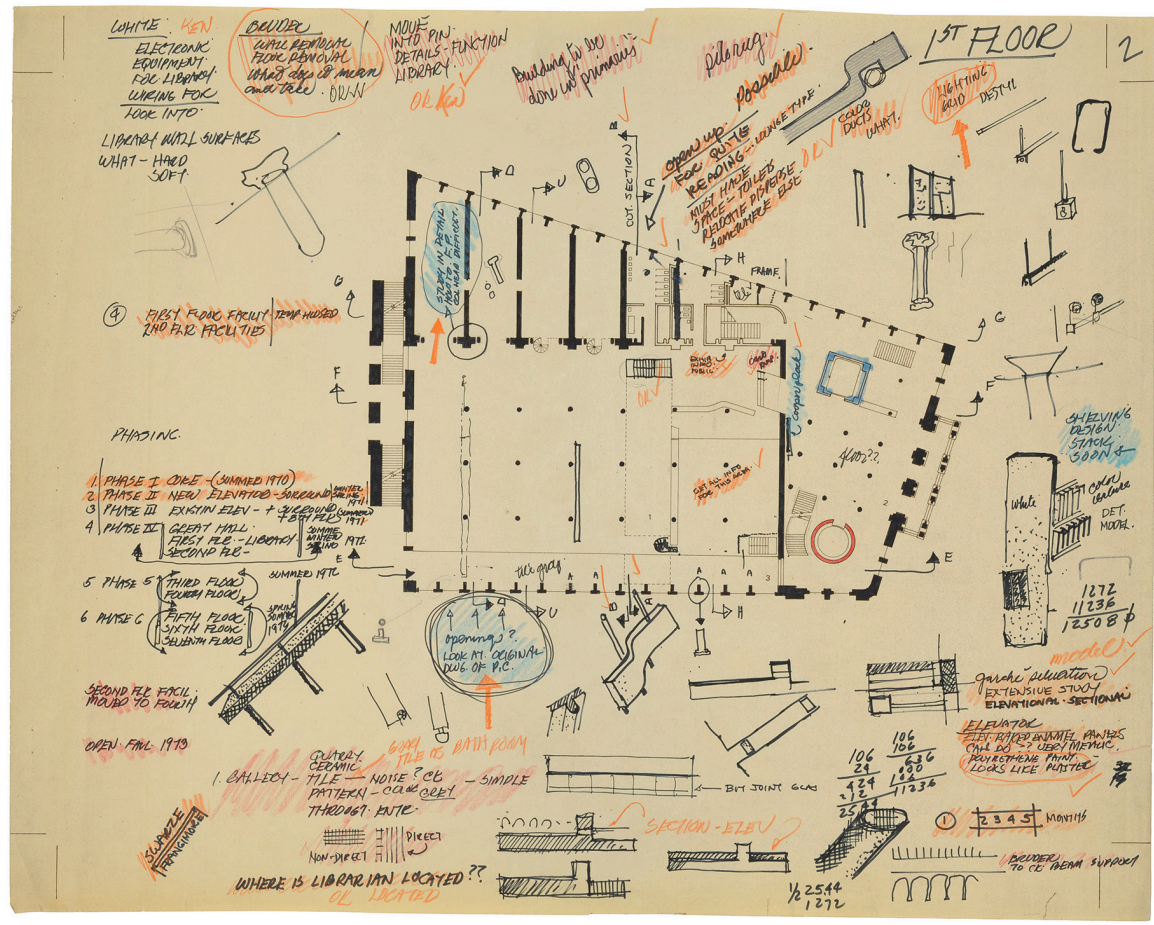

FIGURA 10

TRANSFORMACIÓN INTERIOR DEL EDIFICIO FUNDACIONAL

DE LA COOPER UNION: PLANTA BAJA DEL PROCESO DE DISEÑO

HEJDUK, J. (1972). COOPER UNION FOUNDATION BUILDING INTERIOR RENOVATION, FIRST

FLOOR DESIGN DEVELOPMENT PLAN. [PLANO DE TRABAJO]. NUEVA YORK: CORTESÍA

THE IRWIN S. CHANIN SCHOOL OF ARCHITECTURE ARCHIVE, THE COOPER UNION.

Hejduk al seleccionar el círculo, el cuadrado y el triángulo hace referencia al elementarismo ${ }^{10}$ de Kandinsky de la serie triángulo amarillo, cuadrado rojo y círculo azul que propone identificar una correlación universal entre color y geometría a partir de las "correlaciones entre línea-plano-color" (Kandinsky, 1970, p. 77) . Hejduk por su parte define el problema específico a estudiar a través de la selección, clasificación y codificación de los elementos - forma, color y condición espacial - con los que trabajará dentro de la estructura de los nueve cuadrados. Utiliza el lenguaje visual basado en la idea de la serie de oposiciones perceptivas de Kandinsky pero entendiéndolo como una herramienta para construir el lenguaje arquitectónico de este proyecto en particular.

En la parte inferior central de la planta sótano Hejduk relaciona al círculo rojo con la condición neutra, al cuadrado azul con la compresión y al triángulo amarillo - panel fragmentado en vertical o diagonal - con la expansión, al estar encerrado (Figura 9 y Figura 10). En la planta, los dos volúmenes que contienen los elevadores, el círculo rojo y el cuadrado azul, se contraponen como elementos opuestos y complementarios 
mientras el triángulo amarillo que representa los planos verticales es el elemento que conceptualmente está entre los dos (intermediario).

Al desarrollar el contenido de la relación entre forma y color, la curva del círculo, sin aristas, contiene en sí misma todas las direcciones generando un movimiento continuo y fluido que está representado por el color rojo. Al estudiar esta forma aisladamente, no existe jerarquía en las direcciones ni en los ejes; por lo tanto, Hejduk define al cilindro como un elemento neutro. En contraposición, el cuadrado está delimitado por cuatro aristas y define dos ejes - cuatro direcciones - en relación con su orientación. La estabilidad de estas direcciones está representada con el color azul y es el elemento que Hejduk utiliza para comprimir el espacio.

Acompañando la dialéctica entre el círculo rojo-neutro y el cuadrado azul-comprime, Hejduk dibuja en la parte superior derecha de la planta sótano una serie de planos de color amarillo que corresponden a paneles divididos vertical (rectángulo) o diagonalmente (triángulo) (Figura 9). La leyenda correspondiente dice "expande cuando está encerrado". Si bien es cierto, el triángulo hace una clara referencia al límite vertical de las escaleras que rodean el cilindro y conectan la planta sótano con la planta baja, la estrategia que busca la expansión del espacio al delimitarlo se aplica en todas las plantas de esta zona del edificio porque los planos verticales que configuran los espacios están siempre exentos de la estructura, son elementos independientes y discontinuos en relación con las columnas de planta circular y nunca llegan a formar un volumen, siempre mantienen su carácter de plano. ${ }^{11}$ Con este elemento, cierra la trilogía de formas y colores entendiendo conceptualmente al triángulo amarillo como un plano fragmentado del color más cercano a la luz, el amarillo, porque busca la expansión del espacio. 

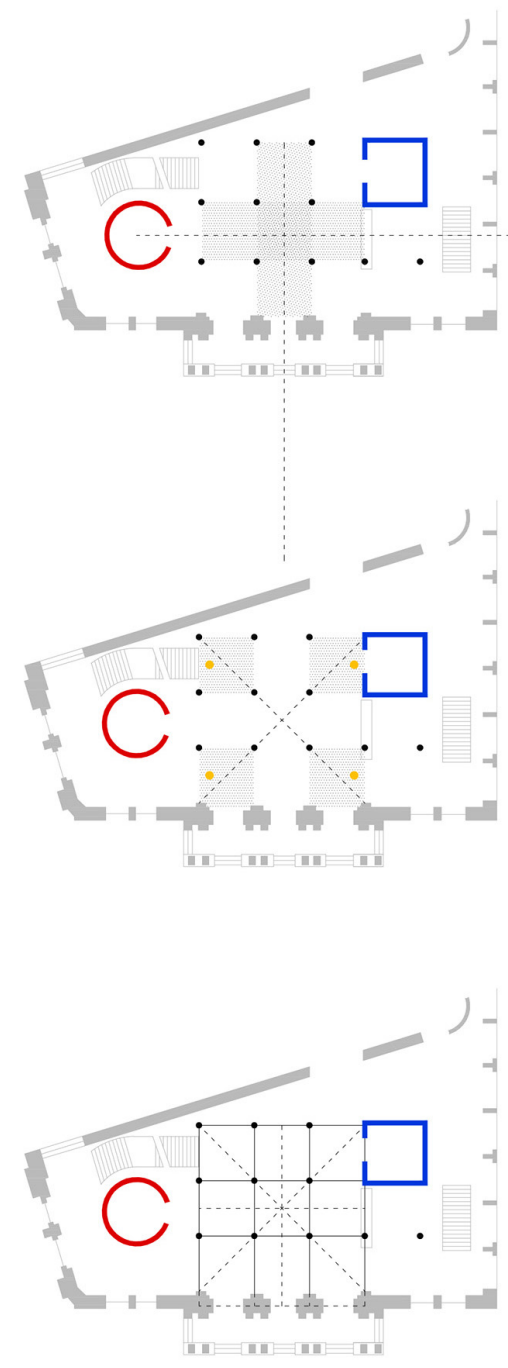

FIGURA 11

ANÁLISIS DEL CONCEPTO CENTRO-PERIFERIA DEL VESTÍBULO DE LA COOPER UNION ELABORACIÓN PROPIA. (2019)

El diálogo entre el círculo y el cuadrado se produce a través de los nueve cuadrados del vestíbulo. El círculo que en abstracto contiene todas las direcciones, en relación con los nueve cuadrados en el proyecto original de 1859 adopta una dirección predominante al estar relacionado con uno de sus ejes centrales, y Hejduk en la transformación lo mantiene exento de la estructura portante de los nueve cuadrados. Por su parte, el cuadrado del nuevo elevador se dispone en la posición más coherente con su forma, en la esquina nor-este donde delimita con un ángulo recto el espacio de los nueve cuadrados - vestíbulo - con los dos ejes que su forma determina integrándose en un solo elemento con la estructura portante. En términos compositivos, la no coincidencia de los ejes entre círculo y el cuadrado genera una tensión espacial en diagonal donde el círculo es el origen de un movimiento centrífugo, mientras el cuadrado comprime el espacio y conduce el movimiento.

Hejduk construye el lenguaje y la lógica interna del proyecto de forma gradual a partir de la comprensión de los elementos - círculo, cuadrado, triángulo - del problema específico del proyecto. La definición de la condición espacial del círculo - cuadrado - triángulo está relacionada con los dos polos opuestos que plantea en el problema de los nueve cuadrados: "uno de fluidez completa y uno de contención completa" (Hejduk, 
1985, 38), fluidez y movimiento acompañando a la zona del círculo y compresión y delimitación en la zona del cuadrado.

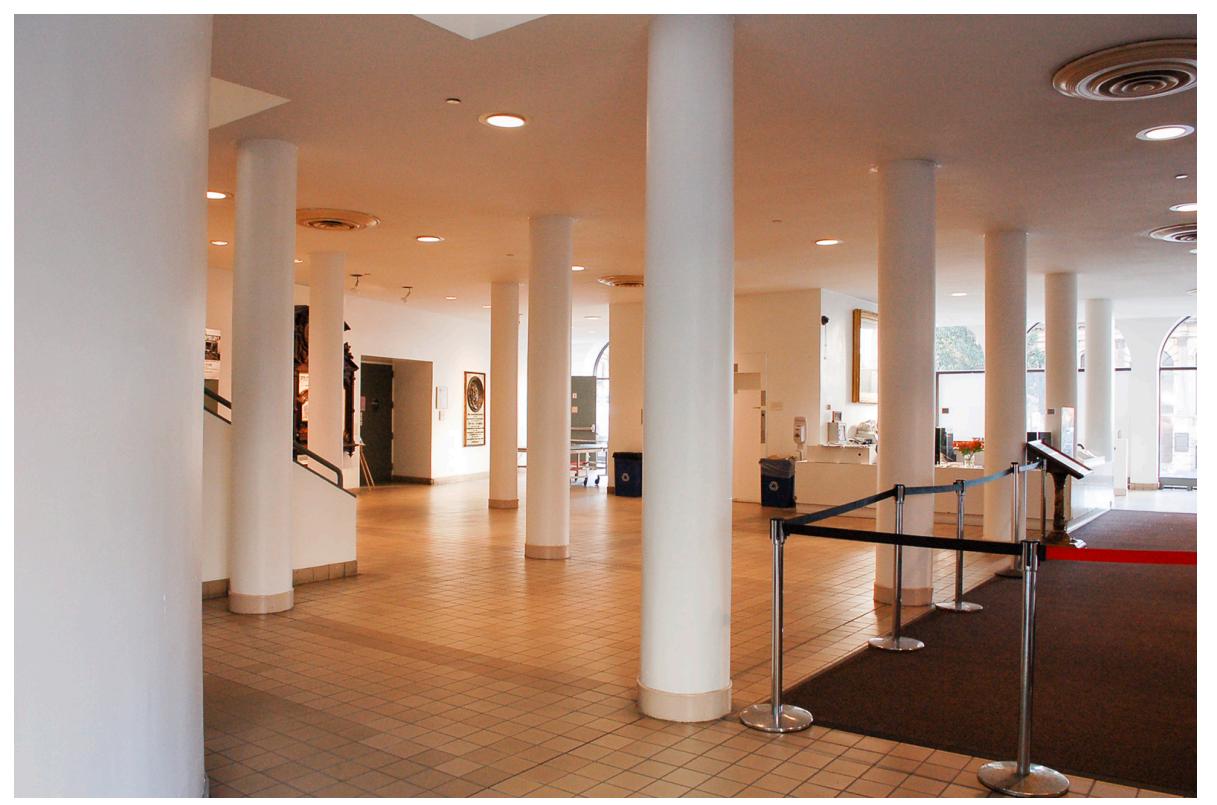

FIGURA 12

FOTOGRAFÍA DESDE LA ESQUINA SUROESTE DE LA PLANTA BAJA DEL VESTÍBULO DE LA COOPER UNION. EN PRIMER PLANO ESTÁ LA CURVA DEL CILINDRO DEL ELEVADOR. ELABORACIÓN PROPIA. (2019)

Para McNeur (1983) la condición centro-periferia en los nueve cuadrados puede ser interpretada de muchas formas. Algunas de ellas son: un centro con una periferia de espacios, estableciendo una relación ortogonal o una relación esquina-diagonal. En la renovación del edificio, Hejduk define la relación ortogonal superponiendo al eje de acceso frontal un eje perpendicular que inicia en el círculo del elevador y se extiende visualmente hacia el exterior. Establece la relación esquina-diagonal definiendo un punto de entrada al vestíbulo de los nueve cuadrados en cada una de sus esquinas (Figura 11). Con esta acción hace una referencia clara a Le Corbusier. Moneo (1980) explica que Hejduk utiliza "el movimiento como un parámetro para estructurar el espacio." Este procedimiento "se comporta como un conjunto inusual de co-ordenadas que dan significación a la arquitectura al situarla en ejes espacio-temporales, a los que uno debe referirse en términos de posición (espacio) y en términos de distancia (tiempo)” (p. 79). ${ }^{12}$ En este proyecto en particular, la superposición de los ejes frontal, lateral y diagonales junto con la fuerza centrífuga del círculo en tensión diagonal con el cuadrado disipan la jerarquía del ingreso frontal. Frontalidad y rotación coexisten ${ }^{13}$ y generan un giro multidireccional que hace que el observador cambie su punto de vista constantemente (Figura 12). La renovación del edificio de la Cooper es la manifestación física del concepto de multifrontalidad y está relacionada con la dinámica del movimiento. 

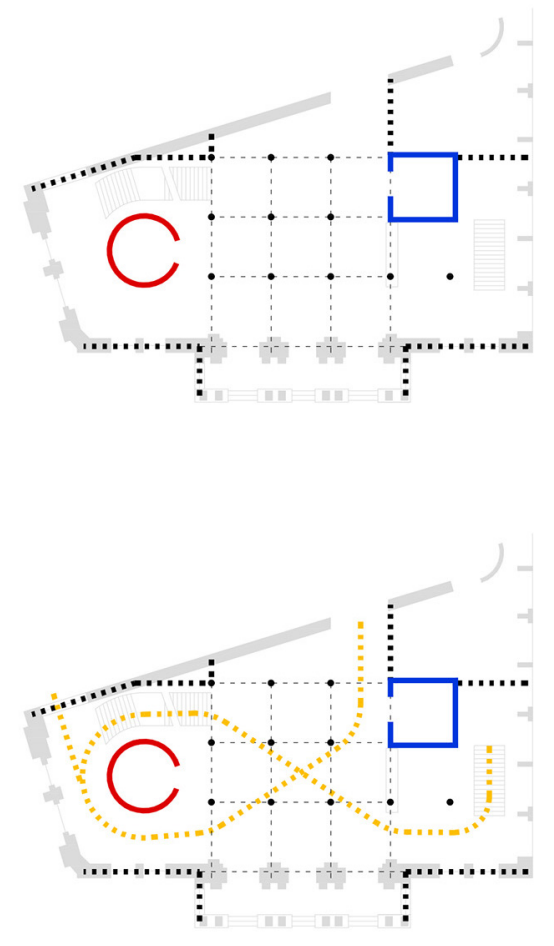

FIGURA 13

ANÁLISIS DE LA RELACIÓN ENTRE NÚMERO Y FORMA DEL VESTÍBULO DE LA COOPER UNION ELABORACIÓN PROPIA. (2019)

Para Moneo (1980), los nueve cuadrados "representan un claro ejercicio de las relaciones que se producen entre número y forma. Es fundamental que el alumno se dé cuenta lo más pronto posible de la diferencia entre cuadrados divididos de distintas maneras, pues su trabajo se basará precisamente en los mecanismos de su división (p. 67). Al estudiar el caso de la transformación de la Cooper Union, la división tripartita de los nueve cuadrados del espacio del vestíbulo se mantiene al analizar la planta completa de esta zona. En este escenario, el vestíbulo se convierte en la celda central de los nueve cuadrados y las ocho celdas de la periferia se conciben de dos formas distintas. Las cuatro celdas contiguas al centro forman espacios de ingreso y las cuatro celdas de las esquinas se desvinculan de éste (Figura 13). El énfasis en posicionar al observador en cada uno de los lados del cuadrado al configurar cuatro ingresos lo más equivalentes posibles considerando las condiciones particulares del proyecto puede estar asociado con la villa Rotonda de Andrea Palladio (1567-1569) donde la aproximación y el ingreso al espacio central es equivalente desde los cuatro lados. El proyecto de Palladio concibe el espacio desde las cuatro direcciones y tiene implícito el concepto de multifrontalidad. ${ }^{14}$ Las coordenadas espacio temporales a las que esta configuración hace referencia posicionan al observador frente a cada uno de los lados del cuadrado central y lo hacen atravesar el espacio sobre el plano horizontal, una sección tras otra en sus cuatro direcciones.

Si bien es cierto, el problema de los nueve cuadrados en esencia es el estudio de la planta, su contenido teórico parte de la comprensión del espacio en arquitectura como el resultado de la superposición de una secuencia temporal de secciones desde los cuatro lados del cuadrado y desde sus diagonales. Esta concepción difiere del énfasis en la frontalidad de Le Corbusier ejemplificado en la Villa Stein y está relacionado con el estudio que hace Hejduk del Carpenter Center en el artículo titulado "Out of Time into Space" ${ }^{15}$ Concepción que tiene su origen en una condición analítica que concibe el espacio a partir de las interrelaciones generadas entre la secuencia de secciones que se producen desde los cuatro lados del telar tridimensional de los nueve 
cuadrados. Didácticamente, una planta cuadrada con todas las secciones con la misma proporción permite que dichas relaciones sean aprehensibles con mayor facilidad. De ahí la importancia de los nueve cuadrados y del cubo como problema para introducir lo que McNeur (1983) denomina "los elementos, órdenes y conceptos de la arquitectura" (p. 24). Prueba fehaciente es que ambos problemas - los nueve cuadrados y las variaciones del problema del cubo - han sido los ejercicios propuestos para el primer año de arquitectura que se han impartido de forma continua en la Cooper Union durante el período en que Hejduk estuvo a cargo de la escuela $(1965-2000){ }^{16}$

\section{Conclusiones}

La importancia que en este proyecto Hejduk da a la definición de todas las direcciones inherentes al cuadrado en relación con la posición del observador cuatro frentes y cuatro diagonales, una desde cada esquina - indica que en realidad lo que estructura el proyecto es el movimiento. Esta premisa se confirma porque Hejduk (1970) explica que la transformación del edificio de la Cooper Union tiene el objetivo de "fomentar el uso por la propia dinámica del espacio arquitectónico" (p. 2). Las coordenadas espacio-temporales que se hicieron evidentes en el análisis de la planta son las que estructuran el movimiento. En el caso de la planta, el espacio se compone en relación con las posiciones del observador (puntos) que el proyecto establece y el tiempo está implícito en la distancia de los ejes que se forman (líneas) sobre el plano base abstracto. El caso de la sección es particular porque coinciden en un solo plano, el plano de la sección, la posición del observador en el espacio y su posición en relación con la distancia: un punto en el tiempo. Esta coincidencia, por un lado, aleja a la sección del plano abstracto de la planta porque representa la mirada del observador que corta el edificio y, por otro, convierte a la sección en un instrumento analítico que permite estudiar simultáneamente los dos conceptos que estructuran el movimiento: espacio y tiempo. Por lo tanto, el movimiento solo se comprende a través de las múltiples secciones que el observador atraviesa en el tiempo desde las posiciones específicas que el proyecto determina. Así, el paso de la planta a la sección es el proceso que permite analizar, comprobar y finalmente integrar de forma coherente lo que en realidad estructura la arquitectura: el movimiento.

Es a través del movimiento que se concibe y produce el paso de la planta a la sección. No es casual que Hejduk en el vestíbulo del edificio de la Cooper Union nos hace atravesar sistemáticamente, como si de un análisis se tratara, sección tras sección desde todas las direcciones inherentes al cuadrado haciéndonos experimentar la relación entre la planta y la sección. Probablemente expresando que la manera de concebir la arquitectura es a través de la comprensión de la lógica interna del proyecto derivada del estudio de las interrelaciones entre las múltiples secciones del proyecto. Este puede ser el punto de partida para comprender el principio detrás del sistema de re-presentación que Hejduk propone en la serie Diamond porque se construye como una proyección tridimensional - axonometría - que pone en relación las re-presentaciones bidimensionales de planta-elevación-sección sin sufrir las distorsiones de otros tipos de axonometrías, manteniendo la verdadera magnitud de los elementos en relación con la posición del observador. En estas condiciones, el recorrido a través de las múltiples secciones del proyecto que esta re-presentación nos invita a hacer con la mirada permite estudiar simultáneamente las variables de espacio y tiempo del proyecto.

Así entendida, la sección tiene un valor instrumental y conceptual de origen analítico. Instrumental porque permite poner en pantalla las relaciones que se producen entre los elementos que componen la arquitectura desde el punto de vista del observador permitiendo valorar las decisiones de proyecto. Conceptual porque permite hacer aprehensible el concepto de tiempo en arquitectura no necesariamente comprendido como una secuencia temporal continua -promenade architecturale-, sino como un elemento a estudiar que intersecado con el espacio está abierto a múltiples posibilidades de configuración.

Ahora, es posible comprender a qué se refiere Hejduk (1971) cuando dice "el alumno empieza a probar el significado de la planta, la elevación, la sección y los detalles. (...) Él empieza a comprender las relaciones entre los dibujos bidimensionales, las proyecciones axonométricas y la forma tridimensional (modelos)” (p. 37). 

EDIF...

No es solamente una compresión operativa y pragmática de las herramientas del arquitecto, sino, además, va acompañada de la comprensión de condiciones intrínsecas de la disciplina: el significado y las implicaciones del paso de la planta a la sección en relación con los conceptos de espacio y tiempo. A este respecto Eisenman (1979) explica que el trabajo de Hejduk en las Texas Houses puede separarse en dos áreas: "primero la manera en la que utiliza los elementos horizontales - el lugar, la planta y el techo - para implicar conceptos del espacio. Segundo, cómo utiliza los elementos verticales - la retícula de columnas y la superficie vertical - para revelar conceptos de tiempo" (p. 10).

No cabe duda de que la renovación del edificio fundacional de la Cooper Union es un extraordinario ejemplo de transformación. Hejduk trabaja en el problema de los nueve cuadrados al ceñirse a las reglas del juego - orden estructural y espacial -estableciendo una relación distinta entre los elementos que inserta dentro de la estructura para así crear "un interior del siglo XX en su caparazón del siglo XIX" (Yee, 1974, p. 96). La superposición de frontalidad y multifrontalidad, de una condición espacial estática con otra que genera movimiento constante, indican que Hejduk introduce otros principios generadores de la forma y del espacio sin eliminar los preexistentes. Para Hejduk (1979) los siete años de trabajo en las Texas Houses justamente fueron la búsqueda de estos principios con la esperanza de expandir un vocabulario. Es su aporte con relación al desarrollo de las ideas del cubismo en arquitectura. Hejduk (1979) escribe: "Fue a través de llevar a cabo estos proyectos que esperé establecer un punto de vista, y la creencia de que, (...) a través de una estética, una liberación de la mente y de la mano pueden ser posibles guiando a ciertas visiones y transformaciones de la forma del espacio" (p. 116). El proyecto de la Cooper Union de Hejduk no es sólo la transformación del edificio, sino, además es la manifestación física del cambio de paradigma "lo estático con lo dinámico: todo esto comienza a tomar la forma de un vocabulario” (Hejduk 1979, p. 116).

\section{Bibliografía}

Anónimo. (1972, septiembre). Renovating a Landmark. At Cooper, 4-7.

Anónimo. (1980). The Passion to Teach. Lotus International, 27, 64.

Crosbie, M. (March 1988). ACSA, AIA Honor John Hejduk For Excellence in Education. Architecture, 19

Eisenman, P. (1979). In My Father's House are Many Mansions. En J. Hejduk, 7 Houses (pp. 8-20). New York: Institute for Architecture and Urban Studies

Frampton, K. (1975). Renovation Cooper Union. Domus, 551, 11-15.

Frampton, K. (1975). Frontalidad frente a Rotación. In Five Architects (pp. 9-13). Barcelona: Gustavo Gili.

Hejduk, J. (1969). Out of Time into Space. Cable The Cooper Union Yearbook, 8-19

Hejduk, J. (1970). The Cooper Union Renovation Project. New York: The Cooper Union.

Hejduk, J. (1971). The Nine-Square Problem. En: The Cooper Union, Education of an Architect: A Point of View (p. 7). New York: The Cooper Union.

Hejduk, J. (1979). John Hejduk: 7 Houses. New York: Institute for Architecture and Urban Studies

Hejduk, J. (1996). John Hejduk. In: Bart Goldhoorn (Ed.), Schools of Architecture (pp. 7-22). Rotterdam: NAi Publishers

Hejduk, J., y Shkapich, K. (Ed.). (1985). Mask of Medusa: Works 1947-1983. New York: Rizzoli.

Kandinsky, V. (1970). Punto y linea sobre el plano. Barcelona: Barral Editores.

McNeur, L. (1983, septiembre). The Nine Square Grid. Section a, 24-27.

Moneo, R. (1980). The work of John Hejduk or the passion to teach. Lotus International 27, 65-81

Moneo, R. (2009). The Four Square Design Problem. Japan Architect, $72-73$

New York Landmarks Preservation Committe. (1966). Cooper Union Foundation Building Designation Report. https://nyclpc.maps.arcgis.com/apps/webappviewer/index.html?id=93a88691 cace4067828bleede432022b 
Scott, T. (2019, noviembre 18). Why the Elevator Shaft Was Invented Before the Elevator [video file]. https://www.y outube.com/watch?v=TL4xc5bsSUU\&t=1s

The Cooper Union. (1970). Undergraduate Curricula 1970-1971. Nueva York: The Cooper Union.

Yee, R. (1974). Metamorphosis. Progressive Architecture, 96-102.

\section{Notas}

* Arquitecto por la Pontificia Universidad Católica del Ecuador (PUCE). Ha sido profesora en el área de proyectos en la PUCE y en la Universidad Central del Ecuador. Ha recibido reconocimientos como alumna y como profesional en concursos públicos de diseño arquitectónico. Obtuvo el título de Máster en Proyectos Arquitectónicos Avanzados en la ETSAM-UPM. Actualmente está continuando sus estudios de doctorado en la misma universidad.

1 Para Hejduk el artículo Out of Time into Space es una derivación del artículo de Colin Rowe y Robert Slutzky Transparency Literal and Phenomenal escrito en el período que compartieron los tres en Texas (ca. 1954-1956).

2 Tanto Rowe (ca. 1973-1974) como Slutzky (ca. 1968-1980) fueron profesores de la Cooper Union en arquitectura. Slutzky fue instructor del problema de los nueve cuadrados.

3 Peter Eisenman (ca. 1973-1983), Rafael Moneo (ca. 1977-1978) y Lorna McNeur (ca. 1976-1977) fueron profesores en la Cooper Union. McNeur hizo el problema de los nueve cuadrados como alumna (1976-1977). A pesar de que ninguno de los tres fueron instructores del problema de los nueve cuadrados en la Cooper Union, el espacio único del taller compartido por los cuatro primeros años de la carrera y la modalidad de críticas abiertas promovían el intercambio.

4 Tiene solo tres escuelas Arte, Arquitectura e Ingeniería más un Departamento de Humanidades.

5 El catálogo de la Cooper Union explica que los estándares de admisión son altos y las plazas limitadas, pues, cada estudiante recibe una beca completa de matrícula.

6 El proyecto fue construido entre 1853-1859, el diseño es de Frederick A. Peterson y tiene alteraciones y adiciones realizadas en 1891 por Leopold Eidlitz.

7 El proyecto completo incluía la renovación del edificio fundacional de 1859 y del edificio de 1912 construido en memoria de Abram S. Hewitt y la integración del edificio de Ingeniería y Ciencia.

8 Hejduk sintetiza cuatro décadas dedicadas a la educación en una conferencia en Holanda en 1995 contando historias, ofreciendo datos puntales de los proyectos y señalando lo que le ha influido.

9 El enunciado del problema contiene una lista sistemática, exhaustiva, y de complejidad creciente de los elementos con los que el alumno estudiará relaciones espaciales dentro de la retícula de los nueve cuadrados (16 columnas). Cada combinación de formas de la lista es un problema. Un resumen de éstos incluye: Paneles completos, $1 / 2$ paneles, paneles curvos-medio círculo, panel completo a 45 o 60 o, 45 - 60, combinar paneles completos $90 \& 45$ / 60, construir escaleras, rampas, construir la estructura del segundo piso y continuar estudiando, tercer piso, construir estructura del techo $1 / 2$ círculo, $1 / 2$ rombo-diamante, $1 / 2$ cuadrado; combinar todos los sistemas.

10 Hejduk trabaja con un sistema similar al relacionar forma-color-programa en la Element house (ca. 1968-1974) que diseña para enseñar arquitectura a su hija Renata cuando era niña.

11 La única excepción a esta regla se produce cuando los planos se intersecan con la envolvente del edificio. En este caso, pierden su independencia y forman un solo cuerpo con ésta.

12 Moneo hace esta observación con relación a la 3\%4 House de Hejduk de 1968.

13 Hejduk junto con Robert Slutzky presentaron su investigación sobre este tema en la exhibición de 1967 The Diamond in Painting and Architecture. La relación de la transformación del edificio de la Cooper con los tres proyectos que Hejduk presentó en la exposición se confirma porque en el plano de trabajo (ca. 1970) de la octava planta del edificio de la Cooper él dibuja en la pared que está frente al emblemático reloj el esquema del "diamond." Véase el catálogo de la exhibición con la serie Three Projects de John Hejduk publicado en 1969 y el artículo Frontalidad frente a Rotación con el que Kenneth Frampton en 1972 cura la obra de los cinco arquitectos de Nueva York.

14 La referencia a Palladio está relacionada con la influencia de Colin Rowe en este período. Específicamente con su artículo Las matemáticas de la vivienda ideal de 1947 y el libro de Rudolf Wittkower Los fundamentos de la arquitectura en la edad del humanismo de 1949.

15 Publicado por primera vez en Architecture d'Aujourd'hui (1965) como homenaje a Le Corbusier el año de su fallecimiento.

16 El problema de los nueve cuadrados fue propuesto en la Cooper Union desde 1964 cuando Hejduk empezó a dar clases allí hasta 1978. Durante los siguientes 22 años son las variaciones del problema del cubo las que se han mantenido constantes, aunque no ha sido el único problema propuesto para el primer año de arquitectura: Architectonics.

\section{BY-NC-SA}

